\title{
ORIGINAL
}

\section{EVALUACIÓN DE LAS PROPIEDADES PSICOMÉTRICAS DE LA VERSIÓN ESPAÑOLA DEL ROTTERDAM SYMPTOM CHECKLIST PARA MEDIR CALIDAD DE VIDA EN PERSONAS CON CÁNCER (*)}

\section{Yolanda Agra (1) y Xavier Badía (2)}

(1) Unidad de Investigación de Atención Primaria. Área 4 Insalud, Madrid.

(2) Institut de Salut Pública de Catalunya. Universidad de Barcelona.

* Este estudio ha sido financiado por el Ministerio de Educación y Ciencia (DEICMT: PB 94-1293).

\section{RESUMEN}

Fundamento: el presente trabajo aborda la validación de la versión española del Rotterdam Symptom Checklist (RSCL) para evaluar la calidad de vida de las personas con cáncer. El objetivo es evaluar las propiedades psicométricas del cuestionario y su equivalencia con la versión original inglesa.

Método: Se cumplimentó el cuestionario por 162 pacientes oncológicos terminales y 59 pacientes sometidos a quimioterapia. Se evaluó su estructura interna a través de análisis factorial confirmatorio en pacientes oncológicos terminales, capacidad discriminante según situación clínica de los mismos, consistencia interna en ambas muestras y sensibilidad a los cambios, repitiendo el cuestionario tres veces a los pacientes sometidos a quimioterapia.

Resultados: Los pacientes terminales presentaron peor puntuación que los sometidos a quimioterapia para todas las escalas excepto la psicológica. El análisis fáctorial mostró una estructura interna similar a la versión original. La consistencia interna fue $\geq 0,70$ para todas las escalas $y \geq 0,50$ para las subescalas excepto la de quimioterapia. Se encontraron diferencias de puntuación $(\mathrm{p}<0,05)$ para todas las subescalas (excepto la psicológica) antes y después del tratamiento con quimioterapia.

Conclusiones: La similitud de las propiedades psicométricas de la versión española del RSCL con la versión original indica su utilidad como medida subjetiva de bienestar en la investigación clínica de personas con cáncer.

Palahras clave: Calidad de vida. Cáncer. Cuestionarios. Validez.

\section{ABSTRACT}

\section{Evaluation of the Psychometric properties of the Spanish version of the Rotterdam Symptom Checklist to assess Quality of Life of Cancer Patients}

Background: In this work the validation of the Spanish version of the Rotterdam Symptom Checklist (RSCL) to assess quality of life in cancer patients has been approached. The main goal of this study is to evaluate the psichometric properties of the questionnaire and its equivalence with the original English questionnaire

Methods: the questionnaire was administered to a sample of 162 terminal cancer patients and 59 cancer patients treated with chemotherapy. The internal structure, was evaluated through a confirmatory factor analysis in TCP, the discriminatory power according to clinical situation of cancer patientes, the internal consistency in both samples and the responsiveness to the changes over time after chemotherapy treatment was evaluated in PQT.

Results: The internal structure of scales was similar in TCP to the original version. The cancer patients showed worse scores than the PQT. The reliability was $\geq 0.70$ for the scales and $\geq 0.50$ for the subscales except chemotherapy subscale. The scores of the scales were different $(\mathrm{p}<0.05)$ before and after treatment, cxcept for the psychological scalc.

Conclusions: These results confirm the usefulness of the spanish version of the RSCL as a subjective measure of wellbeing in cancer patients.

Keywords: Quality of Life. Cancer. Questionnaire. Validity.

\section{INTRODUCCIÓN}

Correspondencia:

Yolanda Agra Varela

Unidad de Investigación Área 4.

Atención Primaria.

Calle José Arcones Gil, 11

28017 Madrid.

Fax: 3413681865 .

Correo electrónico: yolanda.agra@ servicom.es
El tratamiento de las personas con cáncer ha estado dirigido tradicionalmente hacia la prolongación del tiempo de supervivencia y la reducción del tamaño tumoral. Sin embargo, a pesar de los avances tecnológicos en el tratamiento de la enfermedad oncológica, el 
$50 \%$ de los pacientes con cáncer todavía no pueden ser curados ${ }^{2}$. Esta limitación y el hecho de que los tratamientos utilizados tengan efectos secundarios que afectan de modo importante a la vida del paciente han originado, en los últimos años, un interés creciente por medir la calidad de vida mediante estudios de investigación clínica sobre cáncer. Tanto para los clínicos como para los investigadores la medición de la calidad en pacientes con cáncer es una medida útil, junto con los datos clínicos, para evaluar el beneficio de los diferentes tratamientos activos, así como el de los tratamientos de soporte en medicina paliativa.

La medición de la calidad de vida debe realizarse con instrumentos estandarizados, adaptados a la cultura en la que se vayan a utilizar y con adecuadas propiedades psicométricas ${ }^{2}$. En España, el uso de cuestionarios para medir calidad de vida en pacientes con cáncer se ha visto limitado, por no existir, hasta fechas recientes, adaptaciones al español que cumplan las normas actualmente vigentes ${ }^{3}$. Uno de los más importantes cuestionarios disponible en lengua española para medir calidad de vida en pacientes con cáncer es el cuestionario de la EORTC QLQ $\mathrm{C}-30^{4}$, validado preliminarmente en mujeres con cáncer de mama 5 . Sin embargo, la ausencia de datos publicados sobre la validez de la versión española en pacientes con diferentes tipos de tumor y enfermedad avanzada, y la ausencia de recomendaciones explícitas sobre su uso ${ }^{6}$, aconseja la adaptación de otros cuestionarios que hayan mostrado adecuadas propiedades psicométricas en estos casos.

El Rotterdam Symptom Checklist (RSCL) ${ }^{7}$ es un cuestionario originalmente desarrollado en Holanda, para medir el impacto de los síntomas sobre la calidad de vida en pacientes con cáncer que participan en ensayos clínicos. Ha sido adaptado para su uso en diferentes paises ${ }^{8}$ y recientes estudios 10 recomicndan por su adecuada validez y fiabilidad mostrada en pacientes con diferentes tipos de tumores y estadio de enferme- dad $^{9.10}$. Por estas razones y por su facilidad para ser autocumplimentado fue elegido para ser adaptado al español. Los datos de dicha adaptación y validez preliminares en una muestra de pacientes con cáncer terminal han sido publicados previamente ${ }^{11}$.

El objetivo del presente estudio es evaluar la estructura interna, la fiabilidad y la sensibilidad a los cambios en pacientes con diferente grado de enfermedad oncológica.

\section{SUJETOS Y MÉTODOS}

\section{Descripción del cuestionario}

El RSCL es un cuestionario con 39 ítems que se agrupan en cuatro escalas: síntomas físicos (23 ítems), síntomas psíquicos (7), actividades de la vida diaria (8) y calidad de vida global (1). En algunos estudios la escala física ha mostrado estar constituida por cuatro subescalas: fatiga (6 ítems), dolor (5), gastrointestinal (5) y quimioterapia (5). Las respuestas son de tipo Likert con cuatro categorías para todas las escalas, excepto para la de calidad de vida global, que tiene siete. Las escalas y subescalas se transforman en un rango de puntuación de 0 (mejor percepción de salud) a 100 (peor percepción de salud), excepto para la escala de actividad, que puntúa de forma inversa. El RSCL no produce un índice global. Puede ser autocumplimentado o realizado con la ayuda de un entrevistador ${ }^{8}$.

\section{Muestras del estudio}

El presente estudio se realizó en la Unidad de Investigación de Atención Primaria del Área 4 del Insalud de Madrid durante 1997. Para alcanzar sus objetivos se eligieron dos muestras de pacientes, una constituida por pacientes oncológicos terminales (POT), para evaluar la estructura interna del cuestionario, y la otra formada por pacientes con diferentes estadíos de enfermedad oncológica sometidos a quimioterapia (PQT), para evaluar la sensibilidad a los cambios del 
RSCL debida al tratamiento. En ambas muestras se evaluó la consistencia interna del cuestionario.

- Los POT se eligieron consecutivamente a su entrada en el Programa de Cuidados Paliativos (PCP) del Área '2, siempre que tuvieran enfermedad oncológica terminal (claramente documentada y expectativa de vida inferior a seis meses según informe clínico hospitalario), edad igual o superior a 18 años, Test de Pfeiffer ${ }^{13}$ con menos de tres errores (para excluir disfunción cognitiva), Karnofsky Performance Status (KPS) ${ }^{1+}$ $>20$ y consentimiento informado para realizar el cuestionario. Se precisaban al menos de 150 pacientes para realizar el análisis factorial de los 30 ítems referidos a los síntomas. El tamaño muestral fue calculado según los criterios mínimos exigidos para la realización de esta técnica (al menos cinco por ítem) ${ }^{15}$. Los cuestionarios fueron cumplimentados en el domicilio del paciente por una enfermera contratada al efecto y previamente entrenada. Si el paciente no tenía dificultad para leer o escribir el cuestionario lo rellenaba por sí solo (entregándosele, previamente, instrucciones cstandarizadas de cumplimentación). Además se registraron datos sobre el tipo de tumor primario que padecía y KPS.

- La muestra de pacientes sometidos a quimioterapia estuvo formada por personas con cáncer que fueran a recibir quimioterapia, en los siguientes 10 días, en el servicio de oncología del hospital Ramón y Cajal, desde el 1 de enero al 30 de julio de 1997, con edad igual o superior a 18 años, esperanza de vida mayor de seis meses y KPS $>60$. Se excluyó a aquellas personas que hubiesen recibido quimioterapia en los 30 días previos. Teniendo en cuenta que, según otros estudios ${ }^{16}$, se consideraba clínicamente relevante un cambio de al menos 10 puntos en las escalas principales del RSCL (entre las situaciones antes del tratamiento y después del segundo ciclo) y que en un estudio piloto previo ${ }^{11}$ la desviación típica media de las puntuaciones de las es- calas fue de 20,6, era necesario incluir en el estudio 56 pacientes ${ }^{17}$, para un test unilateral con $a=0,05$ y $\beta=0,20$. Los pacientes que cumplian los criterios de inclusión fueron seleccionados consecutivamente a su llegada al servicio de oncología para autocumplimentar tres cuestionarios: el primero en la sala de espera de la consulta, cuando se les informaba que iban a recibir tratamiento, y el segundo y el tercero en el domicilio, al séptimo día del primer ciclo de quimioterapia y al séptimo día del segundo ciclo, respectivamente. Durante la primera consulta se registraba la edad, sexo, KPS, tumor primario, estadio y tipo de tratamiento quimioterápico de cada paciente que formaba parte de la muestra de estudio.

\section{Plan de análisis}

Para evaluar si la estructura interna de la versión española del RSCL era similar a la de la versión original se realizó un análisis factorial confirmatorio con las puntuaciones obtenidas en POT. Como paso previo, se examinó la matriz de correlaciones de todas las puntuaciones de los 30 primeros ítems del cuestionario. Para comprobar el grado de interrelación de las variables se realizó la prueba de esfericidad de Barletl, y el índice Kaiser-Meyer-Olkin (KMO) ${ }^{18}$. Los factores se extrajeron utilizando el método de componentes principales o de máxima verosimilitud. Para facilitar la interpretación de los factores se realizaron rotaciones ortogonales de los mismos. Los criterios seguidos para incluir los ítems en cada dimensión o factor fueron: tener una carga factorial o saturación mayor del $30 \%$ y no tener cargas mayores en otros factores.

Para evaluar la consistencia interna del RSCL en las muestras estudio se midió el $\alpha$ de Cronbach de los ítems de cada dimensión del cuestionario.

Para comprobar la equivalencia de la versión española con la original inglesa se compararon los resultados obtenidos con los re- 
feridos en el estudio de validación de la versión original realizado en una muestra de 478 mujeres con cáncer de mama en estadio II sometidas a quimioterapia.

Para evaluar la sensibilidad del RSCL a los cambios en la percepción de salud producidos por el tratamiento quimioterápico, se analizó la diferencia de puntuación entre los tres cucstionarios (pretratamiento, a los 7 días del primer ciclo y a los 7 días del segundo ciclo) a través de ANOVA de medidas repetidas ${ }^{19}$ y la prueba del tamaño del efecto ${ }^{20}$. Se utilizó la prueba exacta de Friedman ${ }^{21}$ para analizar, en la muestra de PQT, la influencia que las variables sexo, KPS, tipo de tumor y tipo de tratamiento, pudieran tener en las puntuaciones del cuestionario. A efectos de análisis estadístico, los diferentes esquemas de quimioterapia fueron recodificados en dos grupos: tratamiento quimioterápico con alto potencial emético, que incluía platino o más de dos drogas, y con bajo potencial emético que incluía una o dos drogas sin platino.

Para el procesamiento y análisis de los datos se utilizó el paquete estadístico SPSS 6.1.2. para Windows ${ }^{22}$.

Para los ítems con ausencia de datos se siguieron las recomendaciones de los autores del RSCL $^{8}$, es decir, se registró la media de la puntuación de la subescala siempre que el paciente hubiera cumplimentado al menos el 50\% de ítems de la misma.

\section{RFSULTADOS}

Los resultados de este estudio se refieren a los 162 POT que cumplieron los criterios de inclusión de los 270 que entraron ese año en el programa, y a 59 pacientes PQT. Las características sociodemográficas y clínicas de los sujetos de las diferentes muestras se describen en la tabla 1. El tumor más frecuentemente diagnosticado en ellos fue el cáncer de pulmón. Los PQT tenían mejor nivel funcional, según el KPS, que los POT.
El $42 \%$ de los PQT presentaron estadio $4 \mathrm{y}$ todos ellos tenían cáncer de pulmón.

Tabla 1

Características de los pacientes del estudio

\begin{tabular}{|lcccc|}
\hline Características & $\begin{array}{c}P O T \\
(n=162)\end{array}$ & \multicolumn{2}{c|}{$\begin{array}{c}P Q T \\
(n=59)\end{array}$} \\
\hline Edad & & & & \\
$\quad$ (m; DE) & $66.8: 11.9$ & 55,$7 ; 12.3$ \\
Rango & $31-97$ & $22-76$ \\
Sexo & & & & \\
Hombre & 97 & $(60)$ & 36 & $(61)$ \\
Mujeres & 65 & $(40)$ & 23 & $(39)$ \\
KPS & & & & \\
$30-40$ & 30 & $(18.5)$ & 0 & \\
$50-60$ & 82 & $(50.6)$ & 1 & $(1.7)$ \\
$70-80$ & 50 & $(30.9)$ & 33 & $(55.9)$ \\
$90-100$ & 0 & & 25 & $(42.4)$ \\
Tipo de tumor & & & & \\
Pulmón & & & & \\
Mama & 43 & $(26.5)$ & 33 & $(55.9)$ \\
Colon & 13 & $(8.0)$ & 14 & $(23.7)$ \\
Estómago & 12 & $(7.4)$ & 3 & $(3.4)$ \\
Otros & 11 & $(6.8)$ & 3 & $(3.4)$ \\
\hline
\end{tabular}

Datos absolutus (y porcentajes); m: media; DE: desviación Estándar: POT: pacientes oncológicos terminales., PQT: Pacientes en tratamiento con quimioterapia.

El tiempo medio de cumplimentación del cuestionario fue de 15 minutos en POT y de 9 en PQT. El cuestionario fue bien aceptado y autocumplimentado por los sujetos de la muestra, excepto en el caso de POT, ya que el $83 \%$ de los mismos precisó de asistencia de la entrevistadora para su cumplimentación, debido al deterioro de su estado físico. Hubo ausencia de datos en 11 ítems de la muestra de POT. En PQT hubo ausencia de respuesta a tres ítems del primer cuestionario, a quince del segundo y a seis del tercero. Tres pacientes no pudieron completar el tercer cuestionario, dos por ser derivados a cirugía y otro por efectos adversos de la medicación.

Los POT presentaron peor percepción de salud en todas las escalas, excepto en la psicológica, en relación a PQT antes de ser 
sometidos a quimioterapia. Excepto para la escala actividad, que presentó peor puntuación en POT mayores de 70 años, no se encontraron diferencias importantes en las puntuaciones de las subescalas del RSCL, ni por edad ni por sexo. Tampoco se encontraron diferencias de puntuación significativas según KPS, tipo de tumor (pulmón u otros) o tipo de tratamiento (bajo potencial emético o alto potencial emético) en PQT.
En las tablas 2 y 3 se exponen los resultados comparativos del análisis factorial y alfa de Cronbach entre el presente estudio y el llevado a cabo en la versión original inglesa.

El análisis factorial (AF) presentó un índice $\mathrm{KOM}>0,70$ y el test de Bartlett fue estadísticamente significativo $(p<0,001)$. EI modelo mejor interpretable fue el obtenido con el método de extracción de máxima verosimilitud y rotación quartimax. Se identi-

Tabla 2

Análisis factorial de la versión española (y original inglesa) * del RSCL en POT. Las cargas factoriales $<0,30$ han sido borradas $(n=162)$

\begin{tabular}{|c|c|c|c|c|c|}
\hline \multirow{2}{*}{ Items } & \multicolumn{5}{|c|}{ Factores } \\
\hline & Factor 1 & Factor 2 & Factor 3 & Factor 4 & Factor 5 \\
\hline Ánimo deprimido & $0.72(0.65)$ & - & - & - & - \\
\hline Nerviosismo & $0.71(0.72)$ & - & - & - & - \\
\hline Preocupación & $0.68(0.73)$ & 一 & - & - & - \\
\hline Desesperanza por el futuro & $0.62(0.69)$ & - & - & - & - \\
\hline Irritabilidad & $0.50(0.64)$ & - & - & - & - \\
\hline Estado de tensión & $0.49(0.74)$ & - & - & - & - \\
\hline Ansiedad & $0.48(0.79)$ & - & - & - & - \\
\hline Dolor de boca/dolor al tragar & 0.32 & - & - & - & $(0.63)$ \\
\hline Hormigueos en manos y piernas & - & - & - & - & $(0.40)$ \\
\hline Dificultad para dormir & - & - & $(0.50)$ & - & - \\
\hline Vómitos & - & $0.79(0.74)$ & - & - & - \\
\hline Náusea & - & $0.77(0.78)$ & - & - & - \\
\hline Falta de apetito & 一 & $0.47(0.64)$ & - & - & - \\
\hline Acidez/ardor de estómago & - & 0.41 & 一 & - & $(0.46)$ \\
\hline Estreñimiento & - & 0.30 & - & $(0.58)$ & - \\
\hline Falta de fuerzas & 0.31 & - & $0.70(0.65)$ & - & - \\
\hline Cansancio & 一 & - & $0.66(0.63)$ & - & - \\
\hline Dificultad para respirar & - & - & $0.33(0.58)$ & - & - \\
\hline Boca seca & - & - & $0.32(0.44)$ & - & - \\
\hline Dolor lumbar & - & - & - & $0.64(0.71)$ & - \\
\hline Molestias abdominales & - & 0.36 & - & $0.42(0.52)$ & - \\
\hline Dolor muscular & 0.32 & - & - & $0.39(0.62)$ & - \\
\hline Disminución del interés sexual & $(0.46)$ & - & - & - & - \\
\hline Pérdida de pelo & - & - & - & - & $(0.65)$ \\
\hline Diarrea & - & $(0.33)$ & - & - & - \\
\hline Dificultad de concentración & - & - & $(0.54)$ & - & 0.50 \\
\hline Escozor o dolor de ojos & - & - & - & & - \\
\hline Mareos & 0.34 & $0.31(0.44)$ & - & - & 0.43 \\
\hline Dolores de cabeza & - & - & - & $(0.35)$ & 0.30 \\
\hline Escalofríos & - & - & - & $(0.43)$ & 0.31 \\
\hline
\end{tabular}

RSCL: Rotterdam Symptom Checklist. Según la versión original el factor 1 es la escala psicológica, el factor 2 subescala gastrointestinal, factor 3 fatiga, factor + dolor y factor 5 quimioterapia respectivamente.

(") datos correspondientes à una muestra de 478 mujeres con cáncer de mama en estadio II. 
ficaron en el primer factor los siete ítems que «a priori» se consideraban formaban parte de la escala psicológica, según la versión original del RSCL. Los factores 2 al 5 se relacionaban con la escala física. Los ítems «pérdida de pelo» y «diarrea» que fueron respondidos en la categoría «nada» en un $86 \%$ y $94 \%$ respectivamente y el ítem disminución del interés sexual respondido como «mucho» en el $89 \%$ de los casos, presentaron los niveles más bajos de saturación (tabla 2).

Para las escalas, el alfa de Cronbach presentó un rango de 0.76 a 0,91 en ambas muestras y de 0,29 a 0,79 para las subescalas. La consistencia interna más baja la presentó la subescala quimioterapia (tabla 3).

Tabla 3

Consistencia interna ( $\alpha$ de Cronbach) de la versión española del RSCL y de la versión original inglesa *

\begin{tabular}{|c|c|c|c|c|c|}
\hline \multirow{2}{*}{ Subescalas del RSCL } & \multirow{2}{*}{$\begin{array}{c}P O T \\
(n=162)\end{array}$} & \multirow{2}{*}{$\begin{array}{c}\text { Pretratamiento } \\
\text { Segundo rich } \\
(n=59)\end{array}$} & \multicolumn{2}{|c|}{$\begin{array}{c}P Q T \\
\text { Primer ciclo }\end{array}$} & \multirow{2}{*}{$\begin{array}{l}\text { Versión } \\
\text { Original } \\
\{n=478\}\end{array}$} \\
\hline & & & $(n=59)$ & $(n=56)$ & \\
\hline Física & 0,76 & 0,85 & 0,82 & 0,88 & 0.85 \\
\hline Psicológica & 0,80 & 0,88 & 0,87 & 0,91 & 0,90 \\
\hline Actividad & 0,89 & 0,82 & 0,89 & 0,88 & 0,89 \\
\hline Global & - & - & - & - & 一 \\
\hline Fatiga & 0,58 & 0,77 & 0.71 & 0.79 & 0,74 \\
\hline Dolor & 0,54 & 0.68 & 0.63 & 0,67 & 0,81 \\
\hline Gastrointestinal & 0,63 & 0,69 & 0,50 & 0.64 & 0.88 \\
\hline Quimioterapia & 0,29 & 0,48 & 0.34 & 0.56 & 0.52 \\
\hline
\end{tabular}

RSCL: Rotlerdam Symptom Checklist. POT: pacientes oncológicos terminales. PQT: pacientes sometidos a quimiotcrapia. (*) datos correspondientes a una muestra de 478 mujeres con cáncer de mama en estadio II.

Se encontraron diferencias de puntuación entre la situación basal (pretratamiento) y cl primer y segundo ciclo para todas las escalas y subescalas, excepto para la psicológica, que permaneció sin cambios a lo largo del tratamiento. La subescala quimioterapia presentó, además, diferencias entre el pri- mer y segundo ciclo. El tamaño del efecto fuc muy clevado $(>0,80)$ para la cscala física, subescala gastrointestinal y subescala quimioterapia (entre pretratamiento y segundo ciclo) y moderado $(\geq 0,50,<80)$ para el resto, excepto para la escala psicológica, que fue pequeño $(\leq 0,20)$ (tabla 4$)$. 
Tabla 4

Puntuaciones de las escalas y subescalas de la versión española del RSCL antes del tratamiento, después del primer ciclo y después del segundo ciclo de quimioterapia

\begin{tabular}{|c|c|c|c|c|c|c|c|}
\hline \multirow{2}{*}{$R S C L$} & \multirow{2}{*}{$\begin{array}{c}\text { Pretratamiento } \\
(n=59) \\
M ; D E\end{array}$} & \multirow{2}{*}{$\begin{array}{c}\text { Primer ciclo } \\
(n=59) \\
m: D E\end{array}$} & \multirow{2}{*}{$\begin{array}{c}\text { Segundo ciclo } \\
(n=56) \\
m ; D E\end{array}$} & \multicolumn{2}{|c|}{ MANOVA } & \multicolumn{2}{|c|}{$T E$} \\
\hline & & & & $F$ & $p$ & $P / P C$ & $P / S C$ \\
\hline \multicolumn{8}{|l|}{ Escalas: } \\
\hline Física & $15.8 ; 11.9$ & $25.6: 12.8$ & 27. $2: 15.6$ & 12.1 & 0.00 & -0.82 & -0.96 \\
\hline Psicológica & $26.3 ; 20.9$ & $30.8: 22.5$ & $29.4: 25.4$ & 0.6 & 0.51 & -0.21 & -0.15 \\
\hline Actividad & $85.4: 18.4$ & $76.1: 24.3$ & $76.3: 23.5$ & 3.2 & 0.04 & -0.50 & -0.49 \\
\hline Global & $39.5 ; 25.7$ & $52.3 ; 24.5$ & $46.1 ; 24.2$ & 3.8 & 0.02 & -0.50 & -0.26 \\
\hline \multicolumn{8}{|l|}{ Subescalas: } \\
\hline Fatiga & $26.9: 21.5$ & $36.2 ; 18.9$ & $35.8 ; 21.6$ & 3.8 & 0.02 & -0.43 & -0.41 \\
\hline Dolor & $13.1 ; 15.4$ & $22.3 ; 18.8$ & $21.3 ; 19.4$ & 4.7 & 0.01 & -0.60 & -0.53 \\
\hline Gastrointestinal & $7.6: 12.2$ & $23.7 ; 16.1$ & $22.8: 16.9$ & 20.2 & 0.00 & -1.32 & -1.25 \\
\hline Quimioterapia & $7.9 ; 9.6$ & $13.2: 11.9$ & $23.4 ; 17.1$ & 18.9 & 0.00 & -0.55 & -1.60 \\
\hline
\end{tabular}

TE: tamaño del efecto: m: meclia: DF: desviación estin biar: F: F Snedecor; p: significación estadística: P/PC: diferencias entre pre trataniento y primer cicks; P/SC: diferenciats entre pre tratamiento y segundo cicle.

\section{DISCUSIÓN}

La versión española del RSCL había mostrado, en una muestra de POT de un estudio previo ${ }^{11}$, una validez convergente, capacidad discriminante y fiabilidad adecuadas. En el trabajo actual se confirman las adecuadas propiedades psicométricas de esta versión en una muestra más amplia de pacientes con cáncer, en diferentes estados de enfermedad.

La tasa de respuesta a la cumplimentación repetida del cuestionario en PQT fue del $95 \%$, superior a la referida en estudios similares ${ }^{23}$, probablemente debido al hecho de contratar a una entrevistadora que debía recordar a los pacientes la cumplimentación de los cuestionarios. La mayor ausencia de ítems en la muestra de PQT indica, en consonancia con otros estudios ${ }^{24}$, que la cumplimentación de cuestionarios a través de entrevistador produce menor ausencia de datos que la autocumplimentación sin asistencia.

En el análisis factorial los ítems del cuestionario se agrupan en dos dimensiones claramente diferenciadas, una psicológica $y$ otra física. replicando la estructura interna del cuestionario encontrada en otros estu- dios ${ }^{7.25-27}$. Sin embargo, en los mencionados estudios existe una considerable divergencia en cuanto a las subescalas de la dimensión física, de forma que no en todos se han podido replicar las descritas por los autores originales $^{8}$.

Estas diferencias se pueden deber al método de análisis utilizado y a las diferencias de los sujetos incluidos en los diversos estudios. El método de análisis utilizado aquí ha sido similar al usado en los estudios de validación de la versión original, por tanto, las diferencias encontradas hay que atribuirlas, fundamentalmente, a las diversas características clínicas de los sujetos incluidos, como ya había sido descrito previamente ${ }^{28}$.

La estructura interna de las subescalas del RSCL en este estudio, excepto la de quimioterapia, es parecida a la de la versión original. Las subescalas «fatiga» y "gastrointestinal» presentan una estructura interna similar a la versión original, probablemente debido a que los ítems que incluyen representan síntomas frecuentes en este tipo de pacientes ${ }^{29}$. Sin embargo, el ítem «diarrea», debido a su escasa presencia en estos pacientes, no presenta una saturación suficiente como para incluirse en ninguna subescala. 
Algunos ítems de la subescala dolor están más relacionados con otras dimensiones, por lo que su estructura difiere de dicha subescala en la versión original y la subescala quimioterapia no ha podido ser replicada, ya que al tratarse de pacientes no sometidos a este tipo de tratamiento, los ítems que la componen no están relacionados con esta dimensión.

Las puntuaciones de las escalas del RSCL indican, como se esperaba, una peor percepción de salud en los pacientes oncológicos terminales, excepto para la escala psicológica, que no parece tener, en este estudio, capacidad discriminante. Esta similitud podría deberse al hecho de que los PQT, aunque presentan mejor nivel funcional que los POT, manifiestan importante afectación psicológica cuando acuden al servicio de oncología, a causa de la información recibida tanto sobre su enfermedad como sobre el tratamiento necesario para el manejo de la misma ${ }^{30}$.

La similitud de puntuaciones del cuestionario según edad y sexo (excepto en los POT mayores de 70 años) va a favor de que el factor que más condiciona la calidad de vida de los pacientes es su situación clínica, como ya había sido sugerido en otros trabajos ${ }^{31}$.

La consistencia interna de la versión española del RSCL en relación a la de la versión original ha sido similar para las escalas $\mathrm{y}$ algo inferior para las subescalas, pero ha alcanzando los requisitos mínimos, excepto la subescala «quimioterapia», para permitir comparaciones entre grupos ${ }^{18}$. La mayor consistencia interna en la muestra de PQT se debe a una mayor variabilidad en las respuestas dadas por estos pacientes después de la quimioterapia. La baja consistencia interna de la subescala quimioterapia en POT (que puede explicarse porque al menos el $85 \%$ de los sujetos marcaron en los ítems que la componen categorías bajas de respuesta) hace que se desaconseje su uso en este tipo de pacientes.
Una propiedad esencial de los instrumentos de calidad de vida para ser utilizados en ensayos clínicos, es ser sensibles a los cambios en el estado de salud cuando se realizan intervenciones de reconocida eficacia ${ }^{32}$. Las puntuaciones del RSCL obtenidas en los pacientes sometidos a quimioterapia muestran que los síntomas físicos (especialmente, náuseas, vómitos, cansancio, molestias de estómago y pérdida de pelo) empeoran a lo largo de los ciclos. Las puntuaciones de la escala psicológica han permanecido sin cambios a lo largo del tratamiento, por lo cual parece que la percepción de bienestar está más relacionada con la enfermedad y las expectativas del tratamiento que con el efecto de la quimioterapia, como ya se ha descrito en estudios previos ${ }^{23}$.

Las subescalas «gastrointestinal» y «quimioterapia», presentan las mayores diferencias en cuanto a tamaño del efecto, lo cual sugiere su sensibilidad y utilidad en este tipo de pacientes. Esto es lógico, ya que los ítems que incluyen estas subescalas están claramente relacionados con síntomas secundarios a la quimioterapia.

En cuanto a las limitaciones del estudio cabe mencionar que los POT fueron elegidos consecutivamente a su entrada en el programa, lo cual no permite realizar inferencias de los resultados obtenidos al total de los mismos. No obstante, sus características clínicas y sociodemográficas son similares a las de otros pacientes del programa incluidos en anteriores estudios ${ }^{33}$. El diferente modo de cumplimentación del cuestionario pudo condicionar, en parte, las diferencias encontradas, sin embargo, éstas son similares a las referidas en otros trabajos que incluyen pacientes con cáncer en diferente estadio clíni$\mathrm{co}^{8}$, por lo que parece que las diferencias hay que atribuirlas más bien a la situación clínica del paciente que al modo de administración del cuestionario.

En resumen, se puede decir que la versión española del RSCL ha mostrado en este estudio tener unas propiedades psicométricas 
similares a la versión original. Estos datos confirman la utilidad de la versión española del RSCL como medida del bienestar subjetivo en pacientes con cáncer. Todo ello sugiere que la versión española del RSCL tiene las garantías científicas necesarias para permitir su uso en España en estudios de investigación clínica. Sin embargo, para la toma de decisiones individuales los requerimientos psicométricos del cuestionario deberían de ser mayores.

La validez de esta nueva versión debe de complementarse con evaluaciones en poblaciones de comunidades españolas que presenten diferencias culturales respecto a los sujetos incluidos en este estudio.

\section{AGRADECIMIENTOS}

A la Dra. de Haes por su consejo y ayuda.

\section{BIBLIOGRAFÍA}

1. MacDonald N. The interface between oncology and palliative medicine. En: Doyle D, Hanks GWC. MacDonald N. Oxford textbook of palliative medicine. Oxford: Oxford University Press; 1997.p.11-17.

2. Guillemin F, Bombardier C. Beiton D. CrossCultural adaptation of health-related quality of life measures: Literature review and proposed guidelines. J Clin Epidemiol 1993;46:1417-32.

3. Badía X. Sobre la adaptación transcultural de medidas de calidad de vida relacionadas con la salud para su uso en España. Med Clin (Barc) 1995; 105: 56-58.

4. Aaronson N, Ahmedzai S, Bergman B, Bullinger M, Cull A, Duez N et al. The European Organization for Research and Treatment Of Cancer QLQ-C30: a quality of life instrument for use in international clinical trials in oncology. J Natl Cancer Inst 1993: 85: 365-76.

5. Arrarás LI, Illaramendi JJ, Valerdi JJ. El cuestionario de calidad de vida de la EORTC. Estudio estadístico de validación con una muestra española. Revista de psicología y salud 1995; 7 : 1333.
6. Agra Y, Badía X, Gil A. Instrumentos para la medición de la calidad de vida en pacientes con cáncer. Med Clin (Barc) 1998;110: 703-708.

7. de Haes JC, van Knippenberg FC, Neijt JP. Measuring psychological and physical distress in cáncer patients: structure and application of the Rotterdam Symptom Checklist. Br J Cáncer 1990; 62:1034-38.

8. de Haes JC, Olschewski M, Fayers P, Viss MRM, Cull A, Hopwood P et al. Measuring the quality of life of cancer patients with The Rotterdam Symptom Checklist (RSCL): A manual. Northern Centre for Health Care Research $(\mathrm{NCH})$. The Netherlands: University of Groningen. 1996.

9. Ibbotson T, Maguire P, Selby P, Priestman T, Wallace $L$. Screening for anxiety and depresión in cancer patients: the effects of disease nad treatment. Eur J Cancer 1994:30A:37-40.

10. Montazeri A, McEwen J, Gillis C. Quality of life in patients with ovarian cancer: current state of research. Support Care Cancer 1996; 4: 169-79.

11. Agra Y, Badía X. Spanish version of the Rotterdam Symptom Check List: Cross-cultural adaptation and preliminary validity in a sample of terminal cancer patients. Psycho-Oncology 1998;7: 229-39.

12. Insalud Madrid Área 4. Programa de Cuidados Paliativos a Domicilio. Madrid: Dirección de Atención Primaria Área 4 de Madrid, 1993.

13. Pfeiffer E. A short portable mental status questionnaire for the assessment of organic brain deficit in elderly patients. J Am Geriatrics Soc 1975; 23: 433-41.

14. Karnofsky DA, Abelmann WH, Craver IF et al. The use of nitrogen mustards in the palliative treatment of carcinoma. Cancer 1948; 1: 634-56.

15. Gorsuch RL. Factor Analysis. 2 ed. Hillsdale, New Jersey: Lawrence Erlbaum Associates; 1983.

16. Purohit OP, Anthony C, Radstone CR et al. Highdose intravenous pamidronate for metastatic bone pain. Br J Cancer 1994;70: 554-58.

17. Armitage P, Berry G. Estadística para la investigación biomédica. Barcelona: Doyma. 1997.

18. Nunnally JC, Bernstein IJ. Teoría Psicométrica. 3 ed. En español. México: Mcgraw-hill: 1995.

19. Norusis MJ. SPSS Advanced Statistics « 6.1 for Windows». Chicago: SPSS. 1994.

20. Hays RD, Anderson R, Revicki DA. Psychometric evaluation and interpretation of health-related quality of life data. En: Shumaker SA. Berzor RA, 
eds. The International Assessment of Health Related Quality of Life: Theory. Translation Measurement and analysis. Oxford: Rapid Communications of Oxtord Lid: 1995.p. 103-14.

21. Metha CR. Patel NR. SPSS Exact Test «6.1 for Windows». Chicago: SPSS; 1995.

22. SPSS [statistical pack]. Windows Versión 6.1.2. in Spanish. Chicago: Microsoft Corporation; 1995.

23. Ravaioli A, Buda P. Fava C, Paci E, Tononi A, Riva N. Assessment of the RSCL quality of life instrument during chemotherapy in a italian setting. Qual Life Res 1996: 5: 491-95.

24. Fallowfield LJ Quality-ol-life data. Lancet 1996; 348: $421-22$.

25. Watson M. Law M. Maguire P, Robertson B. Greer S, Bliss JM el al. Further development of a quality of life measure for cancer patients: the Rotterdam Symptom Checklist (revised). PsychoOncology 1992; 1:35-44.

26. Chancellor JV, Coylr D, Drummond M. Constructing health state preference values from descriptive quality of life outcomes: Mission impossible? Qual I ife Res 1997; 6:159-68.
27. Whynes DK, Neilson A. Qual Life Res 1997;6:6166.

28. Fayers PM, Hand DJ. Factor analysis, causal indicators and quality of life. Qual Life Res 1997; 6: 139-50.

29. Conill C, Verger E, Henriquez I, Saiz N, Espier M, Lugo F, Garrigos A. Symptom prevalence in the last week of life. J Pain Symptom Manage $1997 ; 14: 328-31$

30. McGarvey EL, Canterbury RJ, Cohen RB. Evidence of Acute Distress Disorder After Diagnosis of Cancer. South Med J 1998: 91 (9): 864-66.

31. Greimel E, Padilla G Grant M, Saunders J. Physical and psychological outcomes in cancer patients: a comparison of different age groups. Proceedings of ASCO 1995; 14: 501 .

32. Deyo RA, Diehr P, Patrick DL. Reproducibility and responsiveness of health status measures: Statistics and strategies for evaluation. Contr Clin Trials 1991;12 (Supl):142S-158S.

33. Agra Y, Sacristán A, González M. Ferrari M. Portugués A. Calvo MJ. Efficacy of senna versus lac tulose in terminal cancer patients treated with opioids. J Pain Symptom Manage 1998; 15:1-7. 